\title{
Anomalous dielectric response of nanoconfined water
}

Cite as: J. Chem. Phys. 154, 044501 (2021); https://doi.org/10.1063/5.0032879

Submitted: 12 October 2020 . Accepted: 30 December 2020 . Published Online: 22 January 2021

(D) Sayantan Mondal, and (Diman Bagchi

\section{COLLECTIONS}

Paper published as part of the special topic on Fluids in Nanopores
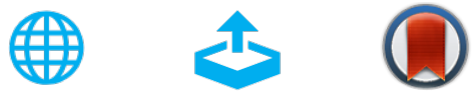

\section{ARTICLES YOU MAY BE INTERESTED IN}

How different are the dynamics of nanoconfined water?

The Journal of Chemical Physics 152, 224707 (2020); https://doi.org/10.1063/5.0010613

A new one-site coarse-grained model for water: Bottom-up many-body projected water (BUMPer). I. General theory and model

The Journal of Chemical Physics 154, 044104 (2021); https://doi.org/10.1063/5.0026651

Dielectric continuum model examination of real-space electrostatic treatments

The Journal of Chemical Physics 154, 044103 (2021); https://doi.org/10.1063/5.0033053

\section{Challenge us.}

What are your needs for periodic signal detection?

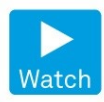

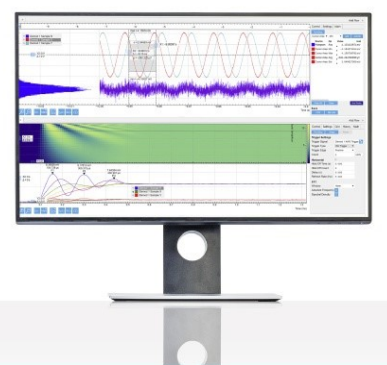

- Zurich

Instruments 


\title{
Anomalous dielectric response of nanoconfined water
}

\author{
Cite as: J. Chem. Phys. 154, 044501 (2021); doi: 10.1063/5.0032879 \\ Submitted: 12 October 2020 - Accepted: 30 December 2020 • \\ Published Online: 22 January 2021
}

Sayantan Mondal (D) and Biman Bagchi ${ }^{\mathrm{a}}$

\begin{abstract}
AFFILIATIONS
Solid State and Structural Chemistry Unit, Indian Institute of Science, Bengaluru, Karnataka 560012 , India
\end{abstract}

Note: This paper is part of the JCP Special Topic on Fluids in Nanopores.

a) Author to whom correspondence should be addressed: b.bagchi@iisc.ac.in

\begin{abstract}
In order to develop a microscopic level understanding of the anomalous dielectric properties of nanoconfined water (NCW), we study and compare three different systems, namely, (i) NCW between parallel graphene sheets (NCW-GSs), (ii) NCW inside graphene covered nanosphere (NCW-Sph), and (iii) a collection of one- and two-dimensional constrained Ising spins with fixed orientations at the termini. We evaluate the dielectric constant and study the scaling of $\varepsilon$ with size by using linear response theory and computer simulations. We find that the perpendicular component remains anomalously low at smaller inter-plate separations $(d)$ over a relatively wide range of $d$. For NCW-Sph, we could evaluate the dielectric constant exactly and again find a low value and a slow convergence to the bulk. To obtain a measure of surface influence into the bulk, we introduce and calculate correlation lengths to find values of $\sim 9 \mathrm{~nm}$ for NCW-GS and $\sim 5 \mathrm{~nm}$ for NCW-Sph, which are surprisingly large, especially for water. We discover that the dipole moment autocorrelations exhibit an unexpected ultrafast decay. We observe the presence of a ubiquitous frequency of $\sim 1000 \mathrm{~cm}^{-1}$, associated only with the perpendicular component for NCW-GS. This (caging) frequency seems to play a pivotal role in controlling both static and dynamic dielectric responses in the perpendicular direction. It disappears with an increase in $d$ in a manner that corroborates with the estimated correlation length. A similar observation is obtained for NCW-Sph. Interestingly, one- and two-dimensional Ising model systems that follow Glauber spin-flip dynamics reproduce the general characteristics.
\end{abstract}

Published under license by AIP Publishing. https://doi.org/10.1063/5.0032879

\section{INTRODUCTION}

Static and dynamic dielectric properties of bulk liquid water are relatively well-understood. ${ }^{1-3}$ In bulk water, the dielectric response is isotropic with a static dielectric constant (SDC) close to 80 under ambient conditions. This large value quantifies the unique ability of water to screen the internal electrostatic interactions and external electric field. Although not fully understood, this large value of the dielectric constant is generally attributed to the collective nature of large dipole moment fluctuations controlled by the extensive hydrogen bond network (HBN) in liquid water. However, this screening ability and the extent of screening could get seriously compromised in a confined geometry. In addition, the response is different in different directions. Given the omnipresence of nanoconfined water (NCW) in biological systems and nanomaterials, it is essential to understand such modulated dielectric properties.
It is well-known that dielectric properties depend on the size and shape of the system. These dependencies become negligible at large system sizes. In recent times, water confined in small volumes draws avid attention because of its importance in biology and materials science. ${ }^{6}$ Earlier theoretical and experimental investigations reveal strong modulation in the structure and dynamics of confined/interfacial water, for example enhanced self-dissociation, diffusion and charge transport, $^{8-14}$ altered phase behavior, ${ }^{15,16}$ viscosity, ${ }^{14,17}$ accelerated reaction kinetics, ${ }^{18,19}$ and solvation and dielectric properties, ${ }^{20-28}$ to name a few. Such studies have given rise to surprising results. However, the microscopic origin of these often observed surprisingly anomalous behaviors has remained elusive.

The alteration of properties of nanoconfined water could arise from three sources. First, the surface-water interactions modify the water layers facing the surface. The influence of this change 
propagates inside. Second, long-wavelength inter-molecular correlations can get truncated so that long-wavelength dynamics, in particular, can get affected. Third, the extensive HBN of water gets distorted. Out of these three effects, only the latter is unique to water. The interaction among these three sources of alteration could give rise to novel effects.

Since surface interactions introduce heterogeneity in the liquid, the description of dielectric properties becomes rather complicated. First and foremost, the dielectric constant becomes position-dependent, and this requires special treatment. ${ }^{23,29}$ Second, the average dielectric constant continues to exist, but the dielectric response becomes anisotropic. ${ }^{20-22,30}$ The theoretical approach becomes non-trivial, as the standard linear response expression needs to be modified, as discussed later.

In an important experimental study, Fumagalli and co-workers measured the out-of-plane (in our terminology, the perpendicular) component of the static dielectric constant (SDC) of water confined between graphene sheets. ${ }^{31}$ They observed a surprisingly small value and an extremely slow convergence of $\varepsilon_{\perp}$ to the bulk with an increasing in inter-slab separation $(d)$. The separation needed to be increased even beyond $100 \mathrm{~nm}$ to recover the bulk value. ${ }^{31,32}$ For smaller systems, the measured value of the out-of-plane dielectric constant was as low as 2. Notably, the surface water layers (approximately two to three molecules thick) become electrically dead, that is, $S D C \sim \varepsilon_{\infty}$. These extraordinary low values at $\mathrm{d} \sim 10 \mathrm{~nm}$ are surprising because we hardly expect effects of the surface to extend so far. In a recent simulation study, Aluru and Motevaselian observed a universal trend in the dependence of $\varepsilon_{\perp}$ on $d$ for several dipolar liquids such as water. ${ }^{30}$

The experimental measurement of Geim et al. used the macroscopic relation between the charge $(\mathrm{Q})$, dielectric constant $(\varepsilon)$, and capacitance (C). By necessity, this method needed to employ two conductive surfaces that contain free charges. Charges are known to interact strongly with the charge distribution of water molecules. Thus, the first layer becomes orientationally immobile. Therefore, this capacitance-based approach measures the response of uniquely modified first few layers. Furthermore, the approach is valid for a large macroscopic system where the heterogeneity in the liquid caused by the surface is negligible. It would be of interest to search for an appropriately extended capacitance relation that reproduces an effective dielectric constant through a self-consistent approach.

In a recent theoretical study, Jalali et al. reproduced the trend in $\varepsilon_{\perp}$, as obtained by Geim and co-workers. ${ }^{33}$ They have reported similar low values of $\varepsilon_{\perp}$ for $\mathrm{d}<1.5 \mathrm{~nm}$ from atomistic simulations of slab-confined water and attributed them to an increase in the number of hydrogen bonds under narrow confinements. However, for systems beyond $\mathrm{d}=1.5 \mathrm{~nm}$, they have employed a meanfield capacitor model. Hence, they have calculated the values of $\varepsilon_{\perp}$ for larger systems from the inputs derived from the smaller systems. Thus, the coherent microscopic understanding and description are presently lacking for this surprising phenomenon. Our present work attempts to provide a microscopic explanation of the experimentally observed anomalous dielectric properties of slabconfined water. In particular, we present an alternative explanation of the low values of SDC in terms of a surface-induced caging potential that quenches dipole moment fluctuations in the perpendicular directions.
A multitude of factors determine the dielectric behavior of confined water. First, the relative strength of intermolecular interactions includes both liquid-liquid and liquid-surface interactions [Eq. (1)]. The latter depends on the hydrophobic/hydrophilic nature of the surface, which plays an important role. ${ }^{34,35}$ Because of the interference with the extensive hydrogen bonding of water, it is hard to quantify the effects of these surface-water interactions. Second, these surface-water interaction effects depend on the shape and the size of the geometric confinement. This is because the surface interactions propagate inward and interfere with others from surfaces on the opposite side, and this could happen differently for different shapes. Third, the confinement may create a bias for certain specific configurations of the liquid molecules in the confined system. That is, some configurations of the system might be preferred over others because of the presence of the surface. When the size of the conferment becomes much larger than the molecular diameter, these effects should become weaker, and the properties are expected to converge to the respective bulk values. This happens when the intermediate and bulk-like internal water layers dominate over the surface layers, as discussed in the context of water inside reverse micelles. ${ }^{36}$ However, for sizes that are not too much larger than the diameter of a water molecule, the HBN of water could get profoundly modified by surface interactions.

The Hamiltonian of the confined water system is given by the following equation:

$$
H=E_{K}^{0}+\sum_{i, j(>i)} u_{i j}(\mathbf{r})+\sum_{i, k} u_{i k}^{\prime}\left(\mathbf{r}^{\prime}\right) .
$$

Here, $E_{K}^{0}$ is the kinetic energy, $u_{i j}(\mathbf{r})$ is the potential energy due to the interaction between $i$ th and $j$ th water molecules, and $u_{i k}^{\prime}\left(\mathbf{r}^{\prime}\right)$ is the potential energy due to the interaction of the $i$ th water molecule with the $k$ th surface atom. The latter needs to be modelled differently to describe different surfaces. It is because of the surface-induced effects one observes several anomalous and puzzling physicochemical properties of confined water.

Several theoretical studies have already explored the spatially resolved dielectric profiles $\left[\varepsilon_{\alpha}(r)\right]$ for different components ( $\alpha=$ radial, parallel, perpendicular)..$^{23,28,29,37}$ Others focused on the effective macroscopic dielectric constant $\left(\varepsilon_{\text {eff }}\right)$ for similar systems. ${ }^{20-22}$ In a remarkable experimental study, Sugahara and co-workers have shown the overscreening of an external field by strongly confined aqueous electrolytic solutions under titanium carbide nano-sheets slit pores. ${ }^{38}$

We note that a significant merit of obtaining d-dependent $\varepsilon_{e f f}$ theoretically is the possibility of direct experimental validation. In addition to this, phenomenological theories of solvation and charge transfer often employ a continuum model based $\varepsilon_{e f f}$ in theoretical discussions of these processes. ${ }^{39,40}$ In this paper, we not only investigate the dielectric properties of nanoconfined water but also introduce and study models of one- and two-dimensional confined Ising spin systems to understand the role of HBN of water. This also allows us to separate the combined effects of surface interactions and confinement. 


\section{THEORETICAL FORMALISM}

According to the theory of macroscopic dielectrics, the components of the dielectric permittivity tensor $\left(\varepsilon_{\alpha \beta}\right)$ are related to the macroscopic polarization (P) and the Maxwell electric field (E). The polarization vector $\mathbf{P}$ is created inside the system in response to the applied E. $\varepsilon_{\alpha \beta}=1+4 \pi\left[P_{\alpha} .\left(1 / E_{\beta}\right)\right]$, where $P_{\alpha}$ and $E_{\beta}$ are the vector components of $\mathbf{P}$ and $\mathbf{E}$, respectively. In an isotropic system (for example, bulk liquid), the diagonal values of the dielectric permittivity tensor are equal, that is, $\varepsilon_{x x}=\varepsilon_{y y}=\varepsilon_{z z}$. In treatments of isotropic liquids, one often employs Kubo's linear response theory (LRT) to obtain the fluctuation formula $(\boldsymbol{P} / \boldsymbol{E})=\left\langle\delta M^{2}\right\rangle / 3 k_{B} T V$, where $\left\langle\delta M^{2}\right\rangle$ is the mean square fluctuation of the total dipole moment. ${ }^{42,4}$

For slab confinement, the system is restricted (non-periodic) in one direction (say, Z) and open (periodic) along the other two directions ( $\mathrm{X}$ and $\mathrm{Y}$ ). As a result, we obtain two unique eigenvalues of the dielectric tensor, namely, $\varepsilon_{\|}$(that is, the same as $\varepsilon_{x x}=\varepsilon_{y y}$ ) and $\varepsilon_{\perp}$ (that is, the same as $\varepsilon_{z z}$ ). By considering different boundary conditions in the parallel and perpendicular directions, we obtain the following linear response relations for $\varepsilon_{\|}$and $\varepsilon_{\perp}$ :

$$
\begin{gathered}
\mathcal{E}_{\perp}^{-1}=1-\frac{4 \pi}{V k_{B} T}\left\langle\delta M_{\perp}^{2}\right\rangle, \\
\varepsilon_{\|}=1+\frac{4 \pi}{V k_{B} T}\left\langle\delta M_{\|}^{2}\right\rangle .
\end{gathered}
$$

Here, $M_{\|}^{2}=M_{Z}^{2}$ and $M_{\perp}^{2}=\frac{1}{2}\left(M_{X}^{2}+M_{Y}^{2}\right)$, which we obtain from equilibrium computer simulations. We note that the relation between the Maxwell field (E) and displacement vector (D), that is, $\mathbf{D}_{(\| / \perp)}=\varepsilon_{(\| / \perp)} \mathbf{E}_{(\| / \perp)}$, remains valid for both the components. Hence, $\varepsilon_{\|}$and $\varepsilon_{\perp}$ quantify the extent of screening along the parallel and perpendicular directions, respectively. We provide the detailed derivation of Eqs. (2) and (3) in Sec. S1 of the supplementary material.

Equations (2) and (3) contain a volume term. This volume term requires a special discussion. Unlike macroscopic statistical mechanics where the volume is determined from outside, in the nanoscopic world, it is determined by the nature of surface liquid interactions. Hence, we find out the number density profiles along the $\mathrm{Z}$ direction $[\rho(z)]$ and integrate it numerically to obtain the effective distance of separation $\left[\mathrm{d}_{e f f}\right]$, as shown in the following equation:

$$
N=\int_{-d_{e f f} / 2}^{+d_{e f f} / 2} d z \rho(z)
$$

Hence, the effective volume $\left(V_{\text {eff }}\right)$ becomes $\left(\mathrm{d}_{\text {eff }} \times \mathrm{A}\right)$, where $A$ is the area of each of the confining slabs. We use these effective volumes, which is somewhat less than the macroscopic volume while evaluating dielectric constants. As the separation $(d)$ increases, $\mathrm{V}_{\text {eff }}$ becomes independent of details of intermolecular interactions.
Here, we would like to point out an interesting property of the wavenumber dependent dielectric constant. It is well-known that the dielectric constant becomes anisotropic even in the bulk when one considers the wavenumber $(k)$ dependence. In order to understand this phenomenon, we need to introduce a coordinate system to discuss wavenumber dependence. At finite $k$, the definition of the longitudinal $\left(\varepsilon_{L}\right)$ and transverse $\left(\varepsilon_{T}\right)$ components is different, that is, parallel and perpendicular to the wave vector $\boldsymbol{k}$. In the intermolecular frame, one considers $\boldsymbol{k}$ along the Z-axis. The projections are different due to different two-particle dipolar orientational direct correlation functions, $\mathrm{c}_{110}(\mathrm{k})$ and $\mathrm{c}_{111}(\mathrm{k})$, respectively, ${ }^{1,44}$

$$
\begin{aligned}
& 1-\frac{1}{\varepsilon_{L}}=\frac{\rho_{0}}{4 \pi} c_{110}(k), \\
& \varepsilon_{T}-1=\frac{\rho_{0}}{4 \pi} c_{111}(k) .
\end{aligned}
$$

In the bulk, the longitudinal and perpendicular components converge to the same value at $k=0$. Nevertheless, they are different at a finite wavenumber. In the present case with confined water inside a slab separated by $d$, the two responses approach each other when the separation $d$ tends to infinity. However, this happens in a highly non-trivial manner. We note that this nomenclature for the intermolecular frame should not be confused with that of the propagating electromagnetic wave.

\section{CAPACITOR MODEL}

The capacitor model has been used in several recent theoretical studies to obtain $\varepsilon_{\perp}$ for slab-confined water. ${ }^{30,33,45}$ We shall not use this to evaluate SDC in this paper, but we aim to connect the experimentally measurable capacitance to the polarization fluctuation. Here, one compares the system to a parallel plate capacitor filled with a dielectric material. For such a system, the capacitance $(C)$ is given by the following equation:

$$
C=\varepsilon A / d
$$

Here, $\varepsilon$ is the dielectric constant of the dielectric medium, A is the surface area of the plates, and $d$ is the inter-plate separation. In Eq. (7), $\varepsilon$ is the perpendicular component of the dielectric tensor, $\varepsilon_{\perp}$. $C$ can be obtained by applying a static external field, perpendicular to the plates. The capacitance (C) can also be obtained from Eq. (8) where $\sigma$ is the charge density on the plates/electrodes and $\phi$ is the voltage difference between the two ends of the capacitor,

$$
C=(Q / \phi)=(\sigma A / \phi)
$$

From Eqs. (7) and (8), one can eliminate the surface area A and obtain an expression for $\varepsilon$ as follows: 


$$
\varepsilon=\left(\frac{\sigma d}{\phi}\right)
$$

Thus, by knowing the inter-plate separation (d) and the electrostatic potential of the plates/electrodes (by solving the Poisson equation with appropriate boundary conditions), one can obtain SDC of the confined medium. However, the capacitor model can only be applied for a non-zero external field. To relate the capacitance with polarization fluctuation, we combine Eqs. (2) and (7). This yields the following equation:

$$
\frac{1}{C}=\frac{d}{A}\left(1-\frac{4 \pi}{V k_{B} T}\left\langle\delta M_{\perp}^{2}\right\rangle\right)
$$

Equation (10) provides a relation between the experimentally observable capacitance $(\mathrm{C})$ with the perpendicular component of the mean squared dipole moment fluctuation $\left(\left\langle\delta M_{\perp}^{2}\right\rangle\right)$ obtained from computer simulations, of course in the linear response regime.

Now, we further analyze this model by dividing the capacitor into $\mathrm{N}$ number of grids of equal thickness $(l)$ along the perpendicular direction. Hence, the system becomes a series combination of $\mathrm{N}$ parallel plate capacitors. Therefore, the effective (or resultant) capacitance $(\mathrm{C})$ can be expressed as

$$
\frac{1}{C}=\sum_{i=1}^{N} \frac{1}{C_{i}}
$$

If we apply Eq. (7) for each capacitor, we get

$$
\frac{1}{\varepsilon}\left(\frac{d}{A}\right)=\frac{l}{A} \sum_{i=1}^{N} \frac{1}{\varepsilon_{i}}
$$

Here, $\varepsilon$ indicates the perpendicular component along which the series connection is assumed. $\varepsilon_{i}$ is the perpendicular SDC of the $i$ th grid that introduces the spatial heterogeneity. If we use $d=N l$, we obtain

$$
\frac{1}{\varepsilon}=\frac{1}{N} \sum_{i=1}^{N} \frac{1}{\varepsilon_{i}} .
$$

It is clear from Eq. (13) that the grids with low values of SDC dominate in the effective value of SDC which is the harmonic mean of the grid-wise SDCs. Hence, the electrically dead water layers with vanishingly low SDC contribute more. ${ }^{45,46}$

Now, we aim to convert Eq. (13) into an integral representation. To this aim, we take the limit of $N \rightarrow \infty$ and also $(d / N)=d z$. Therefore,

$$
\frac{1}{\varepsilon}=\frac{1}{d} \int_{0}^{d} d z \frac{1}{\varepsilon(z)}=\frac{1}{V} \int_{0}^{d} A d z \frac{1}{\varepsilon(z)} .
$$

Here, $\varepsilon(z)$ is the local dielectric permittivity or the spatially resolved dielectric profile. Several earlier studies calculated the position-dependent (or grid-wise) dielectric permittivity for nanoconfined water inside a slab geometry. ${ }^{22,30}$ The water layers adjacent to the surface exhibit an enhancement in $\varepsilon_{\|}(z)$ and a significant attenuation in $\varepsilon_{\perp}(z)$. The accurate estimation of $\varepsilon(z)$ requires a sufficiently long trajectory (sometimes in the $\sim \mu$ s order). This is because the dielectric profiles, especially the one in the nonperiodic directions, show extreme sensitivity to the grid-wise dipole moment fluctuations. Here, from the relatively shorter trajectories, we calculate the grid-wise polarization fluctuations and obtain similar information regarding the enhancements and attenuations discussed above. The mean squared polarization fluctuations serve as the precursors to the grid-wise dielectric constants. We provide the grid-wise parallel and perpendicular $\left\langle\delta M^{2}\right\rangle$ for slab and spherical systems in Sec. S3 of the supplementary material.

However, Eq. (7) has no microscopic origin and is derived by assuming Gauss' law to be valid. Now, for a charged plate, Gauss' law is applicable with the following assumptions: (i) The plate possesses a large surface area, (ii) the charge distribution is uniform, and (iii) the dielectric permittivity is homogeneous. These conditions may not be valid for a nanoscopic system. Hence, its applicability in the nanoscale dimensions remains doubtful.

\section{SYSTEM AND SIMULATION DETAILS}

Here, we simulate different confined systems filled with extended simple point charge (SPC/E) water (Fig. 1, inset) with $d=1.0 \mathrm{~nm}, 2.0 \mathrm{~nm}, 3.0 \mathrm{~nm}, 4.0 \mathrm{~nm}, 5.0 \mathrm{~nm}, 7.0 \mathrm{~nm}$, and $10.0 \mathrm{~nm}$ in the NVT ensemble at $300 \mathrm{~K}$. Here, $d$ is the distance of separation between two square slabs (perpendicular to the Z-axis). We choose the dimensions of the slabs as $5 \times 5 \mathrm{~nm}^{2}$ for $d \leq 5 \mathrm{~nm}$ and $12 \times 12 \mathrm{~nm}^{2}$ for $d>5 \mathrm{~nm}$. The number of water molecules in these confined systems is 536, 1448, 2301, 3174, 4097, 32100 , and 47753 , respectively. We use the modified Berendsen thermostat $(\tau$ $=0.1 \mathrm{ps}^{-1}$ ) to maintain the temperature of the system. After initial energy minimization and equilibration, we analyze from $50 \mathrm{~ns}$ long molecular dynamics trajectories. We impose periodic boundary conditions (PBCs) along the $\mathrm{X}$ and $\mathrm{Y}$ directions (parallel to the plane of the slabs) of the confined water systems. Thus, the system becomes confined along the $\mathrm{Z}$ direction and infinite along the other two (X and $\mathrm{Y}$ ) directions. The wall atoms are modelled as the carbon atoms in graphene sheets. ${ }^{20}$ We find out the optimum number of water molecules inside the confinement from a separate set of $10 \mathrm{~ns}$ long NVT simulations where the external water bath is attached. Hence, water molecules can diffuse in and out of the confinement zone. We obtain the time average of the number of water molecules and take that as the optimum number under confinement. This ensures the equality of chemical potential. For bulk water, we separately simulate a box of SPC/E water of dimension $(5 \times 5 \times 5) \mathrm{nm}^{3}$ in the NVT ensemble $(300 \mathrm{~K})$. The molecular dynamics simulations are performed using GROMACS. ${ }^{47}$ 

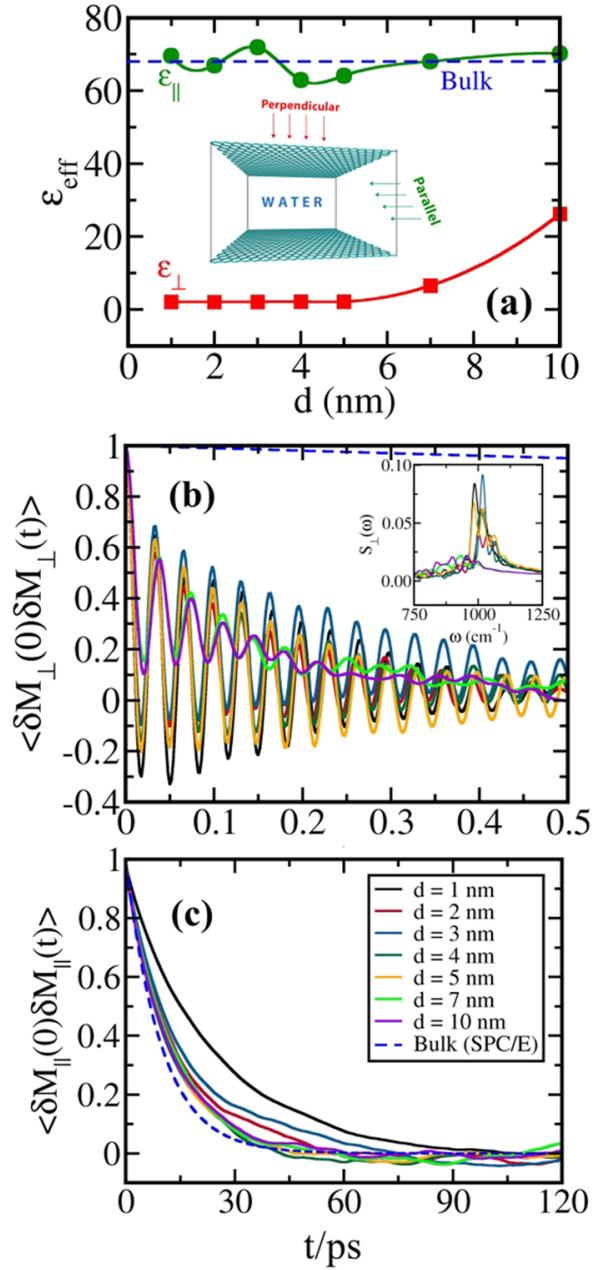

FIG. 1. (a) The parallel $\left(\varepsilon_{\|}\right)$and perpendicular $\left(\varepsilon_{\perp}\right)$ components of the effective macroscopic SDC of water under slab-confinement (as shown in the inset) with an increase in inter-plate distance $(d)$. The parallel component converges rapidly to the bulk value $(\sim 68)$ after the initial oscillatory behavior, whereas the perpendicular component exhibits an extremely slow convergence with an increase in $d$. The surprisingly slow convergence of $\varepsilon_{\perp}$ has also been captured in dielectric microscopy experiments. Relaxation of the (b) perpendicular and (c) parallel components of the total dipole moment time correlation function of slab-confined water. Dielectric relaxation in the parallel direction is slower than the bulk (blue dashed line) and converges to the bulk decay pattern with an increase in inter-plate separation $(d)$. On the contrary, the perpendicular dielectric relaxation exhibits ultrafast and oscillatory relaxation that differs by two orders of magnitude compared to the bulk. Interestingly, all the oscillatory time correlations correspond to a frequency of $\sim 1000 \mathrm{~cm}^{-1}$ [inset, (b)]. As we increase $d$ to $10.0 \mathrm{~nm}$, the oscillation reduces in amplitude, but the decay remains ultrafast.

\section{RESULTS AND DISCUSSIONS}

\section{A. Anisotropic dielectric response of water under slab-confinement}

By using Eqs. (2) and (3), we calculate the perpendicular $\left(\varepsilon_{\perp}\right)$ and parallel $\left(\varepsilon_{\|}\right)$components of the SDC of water under slab-confinement. We depict the results in Fig. 1(a) where we plot $\varepsilon_{\|}$ and $\varepsilon_{\perp}$ against the distance of separation $(d)$ between two hydrophobic plates. We find that the parallel component does not exhibit much difference from the bulk value ( 68 for SPC/E at $300 \mathrm{~K}$ ). However, the perpendicular component exhibits a substantial reduction for the lower values of $d$. We find that $\varepsilon_{\perp}$ shows a slow convergence toward the bulk value. Even at $d=10.0 \mathrm{~nm}, \varepsilon_{\perp}$ is near 40 . This observation, especially for smaller $d$ values, corroborates well with the dielectric microscopy experiments discussed before. ${ }^{31}$ A similar type of slow convergence of the radial component of the SDC with size was reported earlier for cylindrical and spherical nanoconfined systems. ${ }^{2}$

The SDC is the zero frequency value of the real part of the dielectric spectrum. One can obtain the dielectric relaxation spectra by performing an inverse Laplace transform of the total dipole moment correlation function, $\langle\delta M(0) \delta M(t)\rangle$. For anisotropic systems, such as the slab confinement, the dielectric relaxation possesses two components. Hence, in Figs. 1(b) and 1(c), we show the perpendicular $\left[C_{M}^{\perp}(t)=\left\langle\delta M_{\perp}(0) \delta M_{\perp}(t)\right\rangle\right]$ and parallel $\left[C_{M}^{\|}(t)=\left\langle\delta M_{\|}(0) \delta M_{\|}(t)\right\rangle\right]$ components of the total dipole moment relaxation, respectively. We provide the fitting parameters and timescales.

Interestingly, the time correlation function of the perpendicular component of dipole moment fluctuation, $C_{M}^{\perp}(t)$, exhibits ultrafast decay with pronounced oscillations [Fig. 1(b)]. On the contrary, $C_{M}^{\|}(t)$ exhibits slower relaxation compared to the bulk and converges to the bulk pattern with an increase in the size of the confinement [Fig. 1(c)]. The amplitude of the observed oscillations decreases with an increase in $d$, but the timescale of relaxation remains unchanged. We discover the ubiquitous presence of a frequency of $\sim 1000 \mathrm{~cm}^{-1}$ from discrete Fourier transformations of $C_{M}^{\perp}(t)$ [Fig. 1(b), inset]. The frequency is omnipresent for systems with $\mathrm{d} \leq 5 \mathrm{~nm}$ and gradually diminishes for systems with large inter-slab separations. We attribute its origin to the emergence of an effective localization/caging potential in the perpendicular direction.

Gekle and Netz reported such pronounced oscillations in $C_{M}^{\perp}(t)$ for slab-confined water that they connected it to the dominance of librational modes. ${ }^{29}$ For model dipolar liquids, Froltsov and Klapp attributed these oscillations to a dipolaron mode. ${ }^{48}$ In an earlier study, Chandra and Bagchi investigated the existence of dipolaronic modes in dense dipolar liquids. ${ }^{49}$ These modes were observed in the intermediate wavenumbers. Although long-lived collective excitations are challenging to observe in normal liquids due to the insufficient strength of intermolecular interactions and overdamped motions, strong confinement might favor its existence.

\section{B. Correlation length and sensitivity of $\varepsilon_{\perp}$}

In order to search for and to quantify the length scale of spatial correlations, we fit the $\varepsilon_{\perp}$ vs $(1 / \mathrm{d})$ data to a stretched exponential function $a+b \exp (-\xi / d)^{\alpha}$ and extract a correlation length $(\xi)$ of $8.7 \mathrm{~nm}$ along the perpendicular direction with an exponent, $\alpha=3.5$ [Fig. 2(a)]. The presence of such a large correlation length scale justifies the substantially low values of $\varepsilon_{\perp}$ till $\mathrm{d}=7.0 \mathrm{~nm}$. From a similar analysis with experimental data, we obtain the correlation length to be $22.5 \mathrm{~nm}$ with an exponent of 0.75 (Fig. 2, inset). 

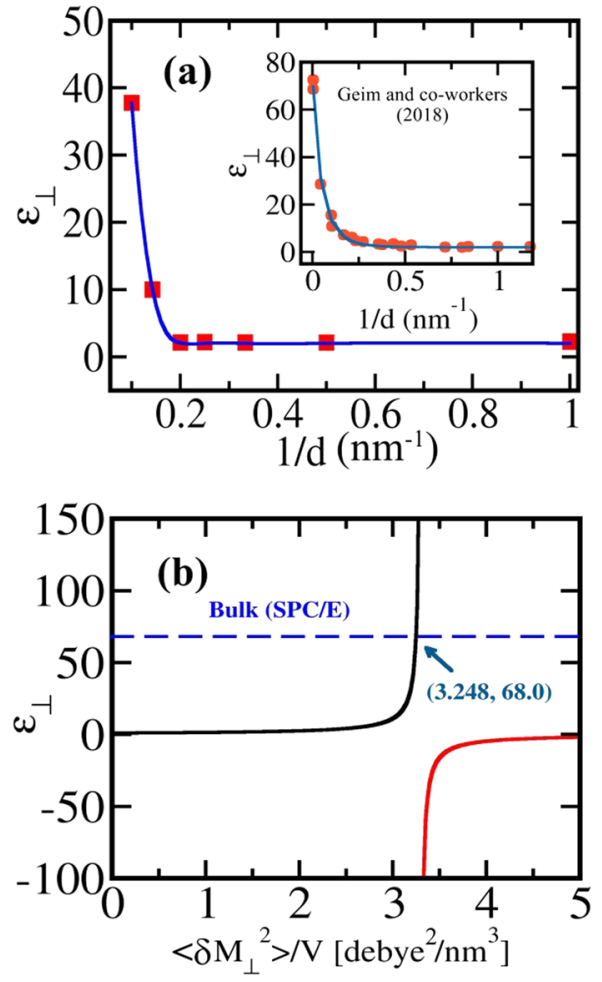

FIG. 2. (a) Plot of $\varepsilon_{\perp}$ against the inverse of the inter-plate separation distance (d). The data are fitted to a stretched exponential form $\left[a+b \exp (-\xi / d)^{\alpha}\right]$. The correlation length $(\xi)$ is found to be $8.7 \mathrm{~nm}$, and the exponent $(\alpha)$ is 3.5 . The parameters $(a=2.0$ and $b=66.0)$ are chosen to reproduce the bulk value (68 at $300 \mathrm{~K}$ ) in the asymptotic limit of $\mathbf{d} \rightarrow \infty$. (Inset) $\varepsilon_{\perp}$ against $1 / \mathrm{d}$ from experimental data that yield $\xi=22.5 \mathrm{~nm}$ and $\alpha=0.75$. (b) The sensitivity of $\varepsilon_{\perp}$ on $\left\langle\delta M_{\perp}^{2}\right\rangle / V$ obtained using Eq. (2) at $\mathrm{T}=300 \mathrm{~K} . \varepsilon_{\perp}$ diverges at around $\left\langle\delta M_{\perp}^{2}\right\rangle / V \sim 3.295$ debye $\mathrm{nm}^{-3}$ and becomes 68 (the bulk value for the SPC/E water model at $300 \mathrm{~K}$ ) for $\left\langle\delta M_{\perp}^{2}\right\rangle / V=3.248$ debye $\mathrm{nm}^{-3}$. A slight deviation from this value could produce either attenuated or highly enhanced values (or even negative values).

While discussing the length scale associated with this problem, one might recall the length scale associated with the hydrophobic force law (HFL). Israelachvili and Pashley obtained a length scale in the order of $100 \mathrm{~nm}$ from experiments. ${ }^{50}$ However, it was shown to be an overestimation later. ${ }^{51,52}$ The true length scale of HFL was surmised to be $\sim 10 \mathrm{~nm}$. It was shown that the long-range effect originated due to the fluctuations and irregularity of the surface. In the present case, the experimental system involves graphene and hexagonal boron nitride plates with polarizability. Besides, the confined liquid is also polarizable. This could give rise to fluctuations different from that of a rigid and non-polarizable graphene-water system.

In Fig. 2(b), we show the sensitivity of $\varepsilon_{\perp}$ to the value of $\left\langle\delta M_{\perp}^{2}\right\rangle / V$. In an earlier study, Chandra and Bagchi showed a similar behavior of the wave-vector dependent dielectric function $[\varepsilon(k)]$ of the dipolar hard-sphere system, although the origin was different.
Here, the origin of the observed sensitivity is purely mathematical and arises due to the use of Eq. (2). $\varepsilon_{\perp}$ starts from 1 for $\left\langle\delta M_{\perp}^{2}\right\rangle / V=0$ and remains low till $\left\langle\delta M_{\perp}^{2}\right\rangle / V=3$ debye $^{2} \mathrm{~nm}^{-3}$. After a certain value of the polarizability, $\varepsilon_{\perp}$ exhibits a sharp rise and diverges before it becomes negative. Because of this ultra-sensitivity, the calculated values of $\varepsilon_{\perp}$ could be highly erroneous, if $\left\langle\delta M_{\perp}^{2}\right\rangle / V$ is not estimated correctly.

The value of the SDC is related to the width of the polarization fluctuations. In Figs. 3(a) and 3(b), we plot the distributions of the parallel and perpendicular components of the total dipole moment per molecule for $\mathrm{d}=1 \mathrm{~nm}$ and $3 \mathrm{~nm}$, respectively. For other systems, we observe a similar pattern. We find that the fluctuations in $\delta M_{\perp}^{2}$ get substantially quenched under confinement, whereas those in $\delta M_{\|}^{2}$ resemble the bulk pattern. Interestingly, the parallel component converges to the bulk value when $4 \pi\left\langle\delta M_{\|}^{2}\right\rangle / V k_{B} T \sim 67$
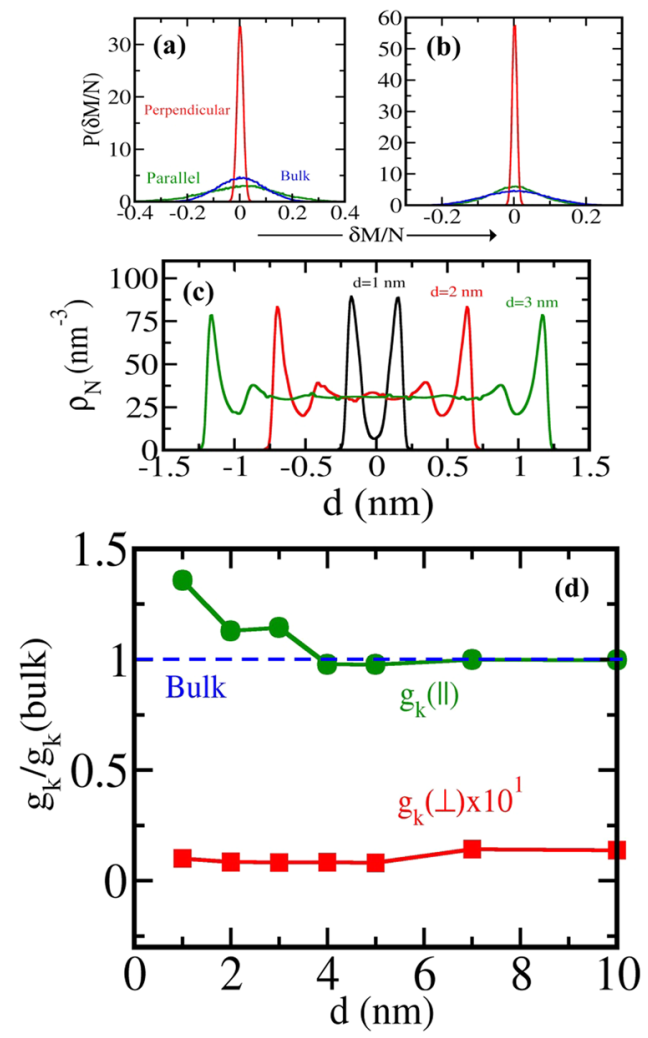

FIG. 3. The distributions of the fluctuations of parallel $\left(M_{\|}\right)$and perpendicular $\left(M_{\perp}\right)$ components of the total dipole moment inside slab nanoconfinement for (a) $d=1.0 \mathrm{~nm}$ and (b) $d=3.0 \mathrm{~nm}$. The distributions of $M_{\|}$(green) show a rapid convergence to the bulk distribution (blue), whereas the distributions of $M_{\perp}$ (red) remain narrow irrespective of the size of the system. (c) Spatially resolved number density profiles of slab-confined water along the perpendicular direction for $d=1.0$ $\mathrm{nm}, 2.0 \mathrm{~nm}$, and $3.0 \mathrm{~nm}$ systems show a multi-layered structure. We obtain similar patterns for the properties shown in (a), (b), and (d) for other systems as well. (d) Components of the Kirkwood g-factor $\left(g_{k}\right)$ with an increase in $d$. The marked difference in the width of the distributions [shown in (a) and (b)] is captured where $g_{K}(\|)$ is two orders of magnitude larger than $g_{K}(\perp)$. 
[Eq. (3)]. On the other hand, the perpendicular component converges to the bulk value when $4 \pi\left\langle\delta M_{\perp}^{2}\right\rangle / V k_{B} T \sim 0.98$ [Eq. (2)]. That is, even a highly quenched fluctuation in $M_{\perp}$ can give rise to the bulk dielectric constant.

\section{Polarization fluctuations and Kirkwood g-factor}

To quantify the intermolecular correlations, we calculate the Kirkwood g-factor $\left(g_{K}\right)$ that is given by the following equation: ${ }^{54}$

$$
g_{K}=\frac{\left\langle M^{2}\right\rangle}{N \mu^{2}} .
$$

Here, $\left\langle M^{2}\right\rangle$ is the total average squared dipole moment, $\mu$ is the single-particle dipole moment (2.35 D for the SPC/E water model), and $N$ is the number of particles. By definition, $g_{K}>1$ indicates the constructive correlation among the water molecules, $g_{K}=1$ indicates the uncorrelated system, and $g_{K}<1$ indicates the destructive correlation. Here, the two parallel hydrophobic surfaces impose opposite orientation on the interfacial water layers that propagate inward. Therefore, the orientations of the water molecules in one half of the system interfere destructively with the orientation of the water molecules in the other half. This aspect can be well-understood in terms of an Ising model described in Sec. VII.

For the slab geometry, we decompose $g_{K}$ into $g_{K}(\|)$ and $g_{K}(\perp)$ [Fig. 3(c)]. We find that the perpendicular component remains more than the bulk value up to $\mathrm{d}=3 \mathrm{~nm}$ and converges to the bulk value by $\mathrm{d}=4 \mathrm{~nm}$. On the other hand, the perpendicular component remains $\sim 50$ times smaller than the bulk for all the systems studied. This indicates a destructive interference of molecular correlations along the perpendicular direction that is attributed to surface effects. In Fig. 3(d), we show the spatial density profiles for $\mathrm{d}=1 \mathrm{~nm}$, $\mathrm{d}=2 \mathrm{~nm}$, and $\mathrm{d}=3 \mathrm{~nm}$ systems. The density profiles are oscillatory and observed for other confined systems such as spherical and cylindrical. As discussed above, we use this density profile to obtain the effective volume by using Eq. (4).

\section{Preferred orientation and quenched rotational dynamics of interfacial water}

In order to develop the microscopic understanding of the problem and its manifestation to the observed anomalies, we calculate the orientation profile of water molecules in the interfacial and internal layers. In Fig. 4(a), we show the distribution of the angles formed by the $\mathrm{O}-\mathrm{H}$ bond vectors with the surface normal $(\theta)$. The distribution is distinct for the water layer at the hydrophobic surface. The interfacial water molecules exhibit a preferential orientation around $\theta \sim 90^{\circ}$. In Figs. 4(b) and 4(c), we show two representative trajectories of rotational jumps of tagged water molecules. We find that the water molecules adjacent to the surface exhibit small amplitude rotations, whereas the distant water molecules show bulk-like large-amplitude rotations. The quenched single-particle dynamics result in a quenched collective dynamics that get manifested in the observed low perpendicular dielectric permittivity for the interfacial layers. As the effective dielectric permittivity is the harmonic mean of the grid-wise dielectric permittivities, the attenuated value of $\varepsilon_{\perp}$ of the surface layers makes a disproportionately large contribution. This eventually reduces the effective value.
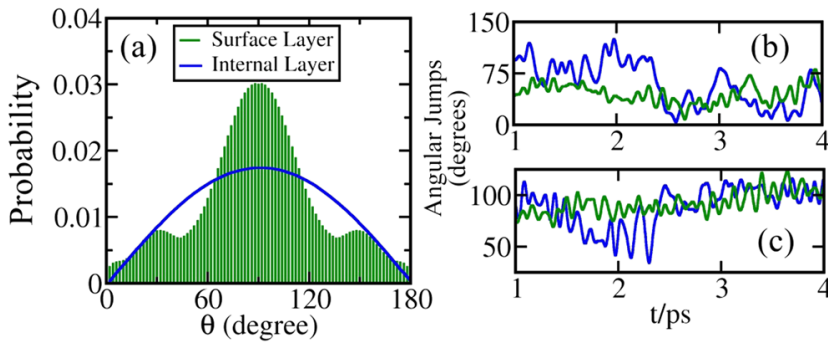

FIG. 4. (a) Distributions of the angles between the $\mathrm{O}-\mathrm{H}$ bond vectors and the surface normal for water molecules $(\theta)$ at the surface (green) and internal (blue) layers. The $\mathrm{O}-\mathrm{H}$ bond vectors of the interfacial water molecules exhibit a preferential orientation at right angles with respect to the surface normal. [(b) and (c)] Representative trajectories of angular jumps of tagged water molecules. The interfacial water molecules exhibit quenched rotational motions (green), whereas the distant water molecules show bulk-like large-amplitude rotational jump motions (blue). The results shown here are obtained for the $d=3.0 \mathrm{~nm}$ slab system.

In order to minimize the free energy of the system and invoke minimum perturbation in the extended hydrogen bond network, water molecules preferentially orient near the surface. This phenomenon is often termed as the principle of minimal frustration. ${ }^{20,21,56}$ This combines with the size and shape of the confining geometry to give rise to the novel behavior. In earlier computer simulation studies, it was found that the water layer closest to the surface is orientationally more rigid (electrically dead) than the water layers inside. ${ }^{2,46,57}$ The same has been observed for the Mercedes-Benz model in contact with hydrophobic rods. ${ }^{56}$ There is evidence of correlated chain structure formation by interfacial and confined water. ${ }^{58-60}$ This kind of surface alignment leads to the inward propagation of correlations and destructive interference.

\section{WATER UNDER SPHERICAL NANOCONFINEMENT}

In order to obtain certain general features and scaling of the SDC, we study the dielectric response of water under spherical nanoconfinements of various radii ranging from $1.0 \mathrm{~nm}$ to $4.0 \mathrm{~nm}$. Unlike parallel slab confinements, the dielectric constant under spherical confinement is isotropic. Hence, one gets a unique value of $\varepsilon_{s}$ by employing the Clausius-Mossotti equation,

$$
\frac{\varepsilon_{s}-1}{\varepsilon_{s}+2}=\frac{4 \pi}{9 V k_{B} T}\left\langle\delta M^{2}\right\rangle .
$$

Here, $\left\langle\delta M^{2}\right\rangle$ is the mean squared total dipole moment of the spherical sample that we obtain from molecular dynamics simulations. We use the same simulation protocols and force field parameters, as described for the parallel slab systems in Sec. IV. The only difference is the absence of periodic boundaries in any direction which makes it an isolated sphere suspended in vacuum. For such systems, Eq. (16) is exactly applicable. 
We plot the SDC of aqueous nanospheres against the inverse of radii ( $R$ ) in Fig. 5(a). The value is low for smaller systems, for example, $\varepsilon_{s} \sim 17$ for $\mathrm{R}=1 \mathrm{~nm}$. We observe a similar slow convergence toward the bulk value with an increase in $\mathrm{R}$, for example, $\varepsilon_{s}$ increases from 17 to 43 when $\mathrm{R}$ increases from $1 \mathrm{~nm}$ to $3 \mathrm{~nm}$. That is, only a 1.5 -fold increase in $\varepsilon_{s}$ for a 26 -fold increase in the sample volume. However, the rate of convergence is faster than nanoslab systems. In order to quantify this, we extract a correlation length scale of $\sim 5 \mathrm{~nm}$ from a stretched exponential plot, as before. This is indeed less than that of the slab systems $(\sim 9 \mathrm{~nm})$. However, it is still quite large compared to the molecular length scale. In Fig. 5(b), we show the total dipole moment autocorrelation functions. The relaxation is ultrafast, and the average timescale is $\sim 20$ times faster than the bulk. We quantify this anomalous behavior of the total dipole moment time correlation function by calculating the Kirkwood g-factor [Eq. (15)]. For all the systems, we obtain $g_{K}$ close to 0.2, which indicate the destructive interference among molecular orientation. We further investigated this and found that the regionwise dipole moments inside a nanosphere strongly anti-correlated.
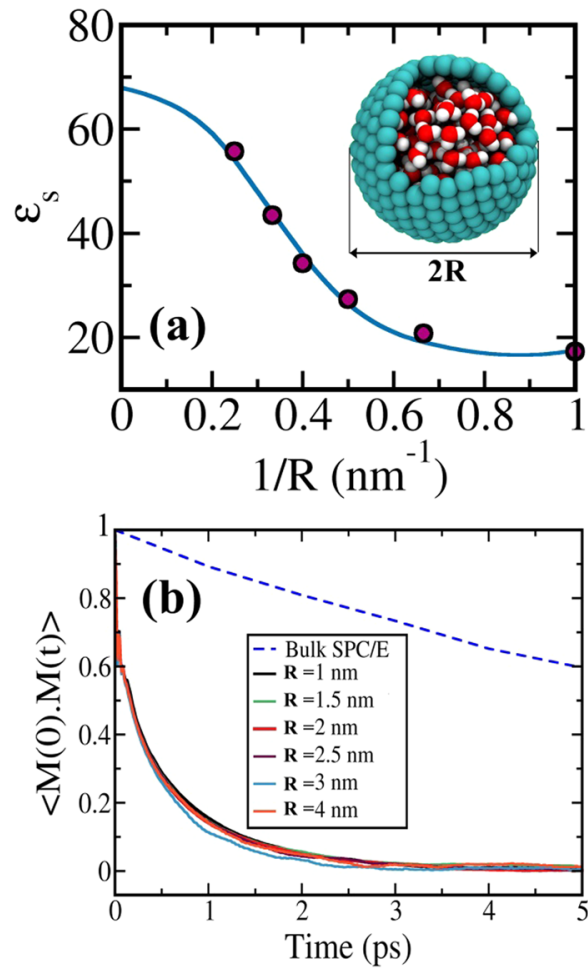

FIG. 5. (a) Static dielectric constant of aqueous nanospheres against the inverse of the radii. The convergence toward the bulk value $(\sim 68)$ is slow. The data are fitted to a stretched exponential form, $a+b \exp \left[-(\xi / R)^{\alpha}\right]$, to obtain a correlation length of $\sim 5 \mathrm{~nm}$ with an exponent of 2.4. The fitting parameters $A$ and $B$ are chosen such that one recovers the bulk value in the $R \rightarrow \infty$ limit. (b) The total dipole moment autocorrelation function of nanospheres of different radii. The relaxation is ultrafast ( 20 times faster than the bulk) and shows no sign of convergence toward the bulk pattern. This could be rationalized by the substantially low values of $g_{K}$ for nano-spherically confined water.
That is, a tremendous cancellation among the coarse-grained dipole vectors occurs.

Therefore, for nano-spherical systems, we obtain an attenuated static dielectric constant, a slow convergence with an increase in radius, and ultrafast total dipole moment relaxations. All these features are similar to the perpendicular component in the case of the slab geometry. This could be a general feature of nanoconfined water. We attribute this behavior to the surface effects and inwardly propagating destructive correlations.

\section{CONSTRAINED ISING SPIN SYSTEMS}

We continue the quest to understand the general characteristics associated with confinement and surface effects. Here, we simulate one- and two-dimensional Ising spin systems without the presence of an external field. ${ }^{55,61}$ For a one-dimensional Ising spin system, the Hamiltonian is given by the following equation:

$$
H_{1 D}=-J \sum_{i=1}^{N-1} \sigma_{i} \sigma_{i+1} .
$$

Similarly, for a two-dimensional system, the Hamiltonian is written as

$$
H_{2 D}=-J \sum_{i, j}\left(\sigma_{i, j} \sigma_{i+1, j}+\sigma_{i, j} \sigma_{i, j+1}\right)
$$

Here, $\sigma_{i}$ is the $i$ th spin in a $1 \mathrm{D}$ system and $\sigma_{i, j}$ is the spin located at the $i$ th row and $j$ th column in a $2 \mathrm{D}$ system. $\sigma$ can assume either +1 or -1 that are assigned randomly to create an initial configuration.

One of the advantages of studying Ising spin systems is the availability of analytical solutions. In a landmark paper, Glauber solved the equation of motion for systems with periodic boundaries and with a fixed central spin. ${ }^{61}$ For the latter, Glauber showed that the equilibrium expectation value for the $k$ th spin (counted from the fixed spin at $\mathrm{k}=0$ ) can be expressed as $q_{k}=\eta^{|k|}$ where $\eta=\tanh \left(J / k_{B} T\right)$. Here, $J$ denotes the strength of coupling between nearest neighbor spins, $k_{B}$ is the Boltzmann constant, and $T$ denotes the temperature. This famous model has been used to understand boundary wall propagation, ${ }^{62}$ frequency-dependent dielectric friction, ${ }^{63}$ and orientation frustration in confined dipolar liquids. ${ }^{55}$ Here, we use a modified Glauber spin system, as detailed below, to understand the scaling of total moment fluctuation with size.

We simulate the confined systems by freezing the orientation of the terminal spins in two ways: (a) both the termini spins are of the same sign and (b) of the opposite sign [Figs. 6(a) and 6(b)]. This is done to mimic the surface-induced orientational rigidity observed for graphene-water systems. For the one-dimensional (1D) systems, we do not apply PBC, and for the two-dimensional (2D) systems, we apply $\mathrm{PBC}$ in one direction. For the bulk system, we perform a set of simulations without any restrictions and $\mathrm{PBC}$ in all the directions that represents the bulk system. $J$ is taken to be $0.6 \mathrm{kcal} / \mathrm{mol}$ for $1 \mathrm{D}$ and $0.3 \mathrm{kcal} / \mathrm{mol}$ for $2 \mathrm{D}$. We keep the temperature of the $1 \mathrm{D}$ systems at $300 \mathrm{~K}$ and that of the $2 \mathrm{D}$ systems at $600 \mathrm{~K}$. We choose a lower value of $J$ and a higher value of $\mathrm{T}$ for the 2D system to avoid any possible 
(a)
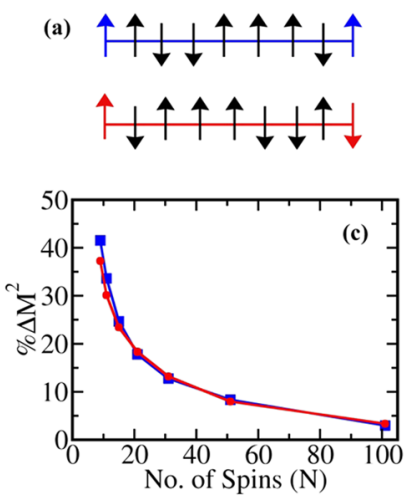

(b)
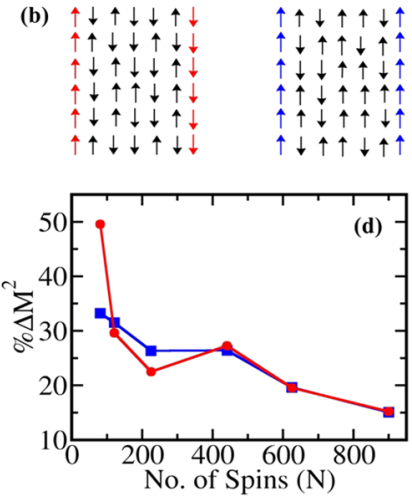

FIG. 6. Schematic representation of the (a) 1D and (b) 2D constrained Ising spin systems. Two types of surface orientations are considered. In one case, the terminal spins possess the same value (shown in blue), and in the other, they possess opposite values (shown in red). The relative reduction in the square dipole moment fluctuations of the confined (c) 1D and (d) 2D Ising spin systems compared to periodic systems of the same dimensions. The difference between $\left\langle\delta M^{2}\right\rangle$ of the periodic and non-periodic system is used as a measure of the difference between the SDCs. The confined spins tend to behave bulk like as the system size is increased.

phase transition. We employ spin-flip Monte Carlo simulation techniques to obtain the mean squared collective spin fluctuation that is related to $\left\langle\delta M^{2}\right\rangle$ in real systems.

In Figs. 6(c) and 6(d), we plot the relative percentage of reduction in the mean squared dipole moments for $1 \mathrm{D}$ and $2 \mathrm{D}$ systems, as defined in the following equation:

$$
\% \Delta M^{2}=\frac{\left\langle\delta M^{2}\right\rangle_{\text {Bulk }}-\left\langle\delta M^{2}\right\rangle_{\text {Confined }}}{\left\langle\delta M^{2}\right\rangle_{\text {Bulk }}} \times 100
$$

For the $1 \mathrm{D}$ spin system, we observe $\sim 30 \%$ to $40 \%$ reduction in the mean squared dipole moment for $\mathrm{N}=9$ and 11 . As we increase the number of spins, $\% \Delta M^{2}$ converges to zero (the bulk limit). The $\% \Delta M^{2}$ vs N plots show a similar behavior and scaling when the orientation of the terminal spins is parallel and anti-parallel. In $2 \mathrm{D}$, we vary the system size from $9 \times 9$ to $30 \times 30$ and find that in the smaller systems, the fluctuations are substantially quenched. We observe that the convergence toward the bulk-like behavior is slower in the $2 \mathrm{D}$ compared to that of $1 \mathrm{D}$.

The simple Glauber spin-flip dynamical models help us to understand certain generalized features of confined dipolar liquids. Here, the rigid surface spins are comparable to the orientationally less mobile water molecules that are adjacent to the graphene carbon atoms. The surface interactions propagate inwardly and its influence diminishes as we move toward the central region. When the dynamics of the central spins (that is, the central layer of water molecules) dominate over the dynamics of the terminal spins (that is, the interfacial water layers), one recovers the bulk-like behavior. Naturally, there arises a correlation length scale that governs the nature of the convergence toward the bulk-like behavior as we obtained for water [Fig. 2(a)]. The correlation length $(\xi)$ is found to be $\sim 0.4 a$ in $1 \mathrm{D}$ and $\sim 33.0 a$ in $2 \mathrm{D}$, where $a$ is the lattice spacing. These values partly explain the slower convergence to the bulk in $2 \mathrm{D}$. The correlation length is, of course, dependent on the strength of coupling $(J)$ between neighboring spins and temperature $(T)$. However, these features seem general and not-too-surprising when one studies such simple model systems.

\section{SUMMARY AND CONCLUSIONS}

Although several theoretical studies exist on dielectric properties of nanoconfined water, relatively less effort has been directed toward a microscopic understanding of the anomalous properties. Furthermore, relatively fewer studies have explored the collective dynamics of such systems. Dynamics could provide an important insight or window into the structure. Thus, the immobility of the first two to three molecular layers of water, adjacent to the surface, catalyzes the progression of an orientational ordering into the deeper regions. This surface-induced ordering seems to be present in all systems consisting of different kinds of molecules, and the extent of influence of the surface seems unusually strong in the case of liquid water because of the hydrogen bonding. Water attempts to preserve its hydrogen bonding network due to enthalpic reasons. The network acquires a different nature, still collective, to accommodate the surface interactions.

One major motivation of the present work is to understand the anomalous results of Fumagalli et al. regarding the abnormally low dielectric constant of confined water. ${ }^{31}$ The present study finds that the alteration and extent of electrostatic screening are markedly different along the parallel and perpendicular directions for aqueous nanoslabs. The anomalously low SDC $\left(\varepsilon_{\perp} \sim 1-2\right)$ along the perpendicular direction is associated with the emergence of a localization potential (or caging potential) with a frequency of about $1000 \mathrm{~cm}^{-1}$. We obtain a correlation length of about $9 \mathrm{~nm}$ that could explain the strong size dependence of $\varepsilon_{\perp}$ and also its low values at smaller $d$ values. We discover a similar behavior for spherically nanoconfined water where the response is isotropic, unlike nanoslabs. Here, we obtain a correlation length of about $5 \mathrm{~nm}$ that is lower than the nanoslab system. This indicates a relatively faster convergence toward the bulk value. However, the value of the correlation length is quite large compared to the molecular diameter. The presence of a large correlation length could be the trademark of nanoconfined dipolar fluids. The investigations on the one- and two-dimensional Ising spin systems help us to understand the finite-size effects and also the influence of the extended hydrogen bonding of water. It shows that an increase in the dimensionality of the system substantially slows down the convergence and increases the correlation length.

We observe, from the present and several earlier studies, at least five general characteristics associated with the dielectric behavior of confined water under nanoslab, nano-spherical, and nanocylindrical geometries. We list them below.

(i) The SDC along the confining direction (that is, along the non-periodic boundaries) is significantly attenuated and exhibits a slow convergence toward the bulk value with an 
increase in size associated with a surprisingly large correlation length. This includes the radial component of SDC for spherical and cylindrical geometries and the perpendicular component of SDC for slab geometries.

(ii) The SDC along the open directions (that is, along the periodic boundaries) is either enhanced ${ }^{21,64}$ or weakly affected by the confinement and exhibits a relatively faster convergence toward the bulk value with an increase in size. This includes the parallel component of SDC for slab geometries and the axial component of SDC for cylindrical geometries. The spherical nano-cavity does not possess such a component.

(iii) The dielectric relaxation along the non-periodic directions is ultrafast, and the total dipole moment fluctuation is severely quenched in the same direction that yields an extremely low value of the Kirkwood g-factor. ${ }^{20,21}$ This could be mapped on to the micro-macro relation discussed by Bagchi and Chandra. ${ }^{63,65}$

(iv) The SDC, along the periodic and the non-periodic directions, is spatially heterogeneous with an oscillatory profile, which makes it difficult to talk about an effective macroscopic SDC. ${ }^{29,37,64,66}$ However, an effective value is required to understand continuum model-based theories and develop implicit solvent-based coarse-grained force fields.

(v) The component of SDC along the non-periodic directions is sensitive to the value of $\left\langle\delta M^{2}\right\rangle / V$ along that direction. In addition to this, $\varepsilon$ exhibits a singularity when plotted against $\left\langle\delta M^{2}\right\rangle / V$. A similar divergent like the behavior of the wavenumber dependent dielectric constant was observed by Chandra and Bagchi for hard spheres by using mean spherical approximation. ${ }^{53}$ Hence, one needs to keep in mind the possibility of large errors in the numerical values of SDC. To avoid this, one must calculate $\left\langle\delta M^{2}\right\rangle$ from a long simulation trajectory and $\mathrm{V}$ numerically from the density profiles.

The question that naturally arises is: What could be the origin(s) of the aforementioned general behavior? We could identify two key factors, which are universally present in these systems: (a) the absence of long wavelength modes and fluctuations associated with those modes along the non-periodic direction and (b) the influence of surface-liquid interaction that partly controls the structure and dynamics of the interfacial water layer that gets induced in the successive water layers. That is, an inwardly propagating surface-induced correlation discussed extensively by Biswas et al. for constrained Ising spins (CISs) and water inside reverse micelles. ${ }^{36,55}$ However, we note that our results are based on nonpolarizable force fields. Hence, the outcomes are solely because of geometric confinement and the chosen liquid-surface interaction parameters.

\section{SUPPLEMENTARY MATERIAL}

The supplementary material contains three sections: (i) the derivations of Eqs. (2) and (3), (ii) error and convergence analyses, and (iii) exemplary plots of polarization profiles for slab and spherical systems.

\section{ACKNOWLEDGMENTS}

This research was supported, in part, by a research grant from SERB (DST), India. S.M. thanks the University Grants Commission, India (UGC), and IISc, Bengaluru, for providing the research fellowship. B.B. thanks SERB (DST) for a National Science Chair professorship for a research grant and financial support. S.M. and B.B. thank the SERC Department of IISc for providing computational facilities (CRAY).

\section{DATA AVAILABILITY}

The data that support the findings of this study are available within the article and its supplementary material.

\section{REFERENCES}

${ }^{1}$ B. Bagchi and A. Chandra, Adv. Chem. Phys. 80, 1-126 (1991).

${ }^{2}$ R. Buchner, J. Barthel, and J. Stauber, Chem. Phys. Lett. 306(1-2), 57-63 (1999).

${ }^{3}$ A. Chandra, D. Wei, and G. N. Patey, J. Chem. Phys. 99(3), 2068-2073 (1993).

${ }^{4}$ B. Bagchi, Water in Biological and Chemical Processes: From Structure and Dynamics to Function (Cambridge University Press, New York, USA, 2013).

${ }^{5}$ P. Ball, Proc. Natl. Acad. Sci. U. S. A. 114(51), 13327-13335 (2017).

${ }^{6}$ S. Mondal, S. Mukherjee, and B. Bagchi, J. Phys. Chem. Lett. 8(19), 4878-4882 (2017).

${ }^{7}$ D. Muñoz-Santiburcio and D. Marx, Phys. Rev. Lett. 119(5), 056002 (2017).

${ }^{8}$ H. Daiguji, Chem. Soc. Rev. 39(3), 901-911 (2010).

${ }^{9}$ G. Hummer, J. C. Rasaiah, and J. P. Noworyta, Nature 414(6860), 188 (2001).

${ }^{10}$ J. C. Rasaiah, S. Garde, and G. Hummer, Annu. Rev. Phys. Chem. 59, 713-740 (2008).

${ }^{11}$ A. Ghoufi, A. Szymczyk, and P. Malfreyt, Sci. Rep. 6, 28518 (2016).

${ }^{12}$ S. Joseph and N. R. Aluru, Nano Lett. 8(2), 452-458 (2008).

${ }^{13}$ R. J. Mashl, S. Joseph, N. R. Aluru, and E. Jakobsson, Nano Lett. 3(5), 589-592 (2003).

${ }^{14}$ C. Sendner, D. Horinek, L. Bocquet, and R. R. Netz, Langmuir 25(18), 10768-10781 (2009).

${ }^{15}$ K. Koga, X. C. Zeng, and H. Tanaka, Phys. Rev. Lett. 79(26), 5262 (1997).

${ }^{16}$ S. Han, M. Y. Choi, P. Kumar, and H. E. Stanley, Nat. Phys. 6(9), 685-689 (2010).

${ }^{17}$ M. Neek-Amal, F. M. Peeters, I. V. Grigorieva, and A. K. Geim, ACS Nano 10(3), 3685-3692 (2016).

${ }^{18}$ Z. Wei, Y. Li, R. G. Cooks, and X. Yan, Annu. Rev. Phys. Chem. 71, 31-51 (2020).

${ }^{19}$ J. K. Lee, S. Banerjee, H. G. Nam, and R. N. Zare, Q. Rev. Biophys. 48(4), 437-444 (2015).

${ }^{20}$ S. Mondal, S. Acharya, and B. Bagchi, Phys. Rev. Res. 1(3), 033145 (2019).

${ }^{21}$ S. Mondal and B. Bagchi, J. Phys. Chem. Lett. 10(20), 6287 (2019).

${ }^{22}$ A. Schlaich, E. W. Knapp, and R. R. Netz, Phys. Rev. Lett. 117(4), 048001 (2016).

${ }^{23}$ V. Ballenegger and J.-P. Hansen, J. Chem. Phys. 122(11), 114711 (2005).

${ }^{24}$ S. Mondal and B. Bagchi, J. Chem. Phys. 152(22), 224707 (2020).

${ }^{25}$ H. Zhu, A. Ghouf, A. Szymczyk, B. Balannec, and D. Morineau, Phys. Rev. Lett. 109(10), 107801 (2012)

${ }^{26}$ R. Renou, A. Szymczyk, and A. Ghoufi, Nanoscale 7(15), 6661-6666 (2015).

${ }^{27}$ C. Zhang, F. Gygi, and G. Galli, J. Phys. Chem. Lett. 4, 2477-2481 (2013).

${ }^{28}$ D. J. Bonthuis, S. Gekle, and R. R. Netz, Phys. Rev. Lett. 107(16), 166102 (2011).

${ }^{29}$ S. Gekle and R. R. Netz, J. Chem. Phys. 137(10), 104704 (2012).

${ }^{30}$ N. R. Aluru and M. H. Motevaselian, ACS Nano 14(10), 12761-12770 (2020). 
${ }^{31}$ L. Fumagalli, A. Esfandiar, R. Fabregas, S. Hu, P. Ares, A. Janardanan, Q. Yang, B. Radha, T. Taniguchi, K. Watanabe, G. Gomila, K. S. Novoselov, and A. K. Geim, Science 360(6395), 1339-1342 (2018).

${ }^{32}$ S. V. Kalinin, Science 360(6395), 1302 (2018).

${ }^{33}$ H. Jalali, H. Ghorbanfekr, I. Hamid, M. Neek-Amal, R. Rashidi, and F. Peeters, Phys. Rev. B 102(2), 022803 (2020).

${ }^{34}$ N. V. Churaev, B. V. Derjaguin, and V. M. Muller, Surface Forces (Springer Science and Business Media, New York, USA, 1987).

${ }^{35} \mathrm{I}$. Brovchenko and A. Oleinikova, Interfacial and Confined Water (Elsevier, Amsterdam, The Netherlands, 2008).

${ }^{36}$ R. Biswas, T. Chakraborti, B. Bagchi, and K. G. Ayappa, J. Chem. Phys. 137(1), 014515 (2012).

${ }^{37}$ C. Schaaf and S. Gekle, J. Chem. Phys. 145(8), 084901 (2016).

${ }^{38}$ A. Sugahara, Y. Ando, S. Kajiyama, K. Yazawa, K. Gotoh, M. Otani, M. Okubo, and A. Yamada, Nat. Commun. 10(1), 850 (2019).

${ }^{39}$ B. Bagchi, Molecular Relaxation in Liquids (Oxford University Press, New York, USA, 2012).

${ }^{40}$ B. Bagchi, Annu. Rev. Phys. Chem. 40(1), 115-141 (1989).

${ }^{41}$ H. Fröhlich, Theory of Dielectrics (Clarendon Press, Oxford, 1949).

${ }^{42} \mathrm{R}$. Kubo, in paper presented at the Statistical Mechanics of Equilibrium and Non-equilibrium, 1965.

${ }^{43}$ R. Kubo, J. Phys. Soc. Jpn. 12, 570-586 (1957).

${ }^{44}$ R. F. Loring and S. Mukamel, J. Chem. Phys. 87(2), 1272-1283 (1987).

${ }^{45}$ C. Zhang, J. Chem. Phys. 148(15), 156101 (2018).

${ }^{46}$ S. Mondal and B. Bagchi, Nano Lett. 20(12), 8959-8964 (2020).

${ }^{47}$ M. J. Abraham, T. Murtola, R. Schulz, S. Páll, J. C. Smith, B. Hess, and E. Lindahl, SoftwareX 1-2, 19-25 (2015).

${ }^{48}$ V. A. Froltsov and S. H. L. Klapp, J. Chem. Phys. 126(11), 114703 (2007).
${ }^{49}$ A. Chandra and B. Bagchi, J. Chem. Phys. 92(11), 6833-6839 (1990).

${ }^{50} \mathrm{~J}$. Israelachvili and R. Pashley, Nature 300(5890), 341-342 (1982).

${ }^{51}$ K. Lum, D. Chandler, and J. D. Weeks, J. Phys. Chem. B 103(22), 4570-4577 (1999).

${ }^{52}$ T. Samanta, R. Biswas, S. Banerjee, and B. Bagchi, J. Chem. Phys. 149(4), 044502 (2018).

${ }^{53}$ A. Chandra and B. Bagchi, J. Chem. Phys. 91(5), 3056-3060 (1989).

${ }^{54}$ S. Saito and I. Ohmine, J. Chem. Phys. 101(7), 6063-6075 (1994).

${ }^{55}$ R. Biswas and B. Bagchi, J. Chem. Phys. 133(8), 084509 (2010).

${ }^{56}$ S. Banerjee, R. S. Singh, and B. Bagchi, J. Chem. Phys. 142(13), 134505 (2015).

${ }^{57}$ S. Ruiz-Barragan, D. Muñoz-Santiburcio, and D. Marx, J. Phys. Chem. Lett. 10(3), 319 (2019).

${ }^{58}$ T. Yamada, S. Tamamori, H. Okuyama, and T. Aruga, Phys. Rev. Lett. 96(3), 036105 (2006).

${ }^{59}$ J. Lee, D. C. Sorescu, X. Deng, and K. D. Jordan, J. Phys. Chem. Lett. 4(1), 53-57 (2013).

${ }^{60}$ M. Nakamura, N. Sato, N. Hoshi, J. M. Soon, and O. Sakata, J. Phys. Chem, C 113(11), 4538-4542 (2009).

${ }^{61}$ R. J. Glauber, J. Math. Phys. 4(2), 294-307 (1963).

${ }^{62} \mathrm{~J}$. Budimir and J. L. Skinner, J. Chem. Phys. 82(11), 5232-5241 (1985).

${ }^{63}$ B. Bagchi and A. Chandra, J. Chem. Phys. 93(3), 1955-1958 (1990).

${ }^{64}$ R. Renou, A. Szymczyk, G. Maurin, P. Malfreyt, and A. Ghoufi, J. Chem. Phys. 142(18), 184706 (2015).

${ }^{65}$ A. Chandra and B. Bagchi, J. Phys. Chem. 94(7), 3152-3156 (1990).

${ }^{66}$ A. Ghoufi, A. Szymczyk, R. Renou, and M. Ding, Europhys. Lett. 99(3), 37008 (2012). 